\title{
EXTREMAL PROBLEMS FOR EIGENVALUES OF MEASURE DIFFERENTIAL EQUATIONS
}

\author{
GANG MENG
}

(Communicated by Yingfei Yi)

\begin{abstract}
Measure differential equations can model non-classical problems like the quantum effects. In this paper we will solve extremal problems for eigenvalues of measure differential equations by exploiting the approximation of general measures by smooth measures and the continuity results of eigenvalues in weak* topology of measures.
\end{abstract}

\section{INTRODUCTION}

Problems linking the coefficient of an operator to the sequence of its eigenvalues are among the most fascinating of mathematical analysis. One of the reasons that makes them so attractive is that the solutions are involved in many different branches of mathematics. Moreover, they are very simple to state and generally hard to solve. For both ordinary and partial differential operators, there have evolved many results [2, 5, 7, 10, 12, 19, 22, In this paper we are interested in extremal problems for eigenvalues of measure differential equations where some physically meaningful measurement is assumed to be fixed.

A basic model in applied sciences is the oscillation of a string which can be described by the second order linear ordinary differential equation

$$
\ddot{y}+q(t) y=0,
$$

where $q \in \mathcal{L}^{1}(J, \mathbb{R})$ with $J=[a, b]$ a finite interval. In this case $q(t)$ can be understood as the density of the string so that the distribution of mass

$$
\mu_{q}(t):=\int_{[a, t]} q(s) \mathrm{d} s, \quad t \in J,
$$

is absolutely continuous (with respect to the Lebesgue measure $\ell$ of $J$ ), while the $\mathcal{L}^{1}$ norm $\|q\|_{1, J}:=\|q\|_{L^{1}(J)}$ can be understood as the "total mass" of the string. Physically, masses of strings may not be absolutely continuously distributed. For example, the Dirac distributions of mass located at one point are even completely singular with respect to the Lebesgue measure. In order to describe the oscillation of these strings, one must extend the theory for ordinary differential equations to measure differential equations, also called generalized ordinary differential equations in some references. Measure differential equations enable us to treat in a unified

Received by the editors January 29, 2013 and, in revised form, July 7, 2013.

2010 Mathematics Subject Classification. Primary 34L15, 34L40.

The author was supported by the National Natural Science Foundation of China (Grant No. 11201471), the Marine Public Welfare Project of China (No. 201105032) and the President Fund of GUCAS. 
way both continuous and discrete systems and have been extensively studied by many authors; see [4, 9, 15,18 .

Let $I=[0,1]$. For a function $\mu: I \rightarrow \mathbb{R}$, the total variation of $\mu$ (over $I$ ) is defined as

$\mathbf{V}(\mu, I):=\sup \left\{\sum_{i=0}^{n-1}\left|\mu\left(t_{i+1}\right)-\mu\left(t_{i}\right)\right|: 0=t_{0}<t_{1}<\cdots<t_{n-1}<t_{n}=1, n \in \mathbb{N}\right\}$.

Let

$$
\mathcal{M}(I, \mathbb{R}):=\{\mu: I \rightarrow \mathbb{R}: \mu(0+) \exists, \mu(t+)=\mu(t) \forall t \in(0,1), \mathbf{V}(\mu, I)<\infty\}
$$

be the space of non-normalized $\mathbb{R}$-valued measures of $I$. Here, for any $t \in[0,1)$, $\mu(t+):=\lim _{s \downarrow t} \mu(s)$ is the right-limit. The space of (normalized) $\mathbb{R}$-valued measures is

$$
\mathcal{M}_{0}(I, \mathbb{R}):=\{\mu \in \mathcal{M}(I, \mathbb{R}): \mu(0+)=0\} .
$$

For simplicity, we write $\mathbf{V}(\mu, I)$ as $\|\mu\|_{\mathbf{V}}$. By the Riesz representation theorem [1], $\left(\mathcal{M}_{0}(I, \mathbb{R}),\|\cdot\|_{\mathbf{V}}\right)$ is the same as the dual space of the $\operatorname{Banach}$ space $\left(\mathcal{C}(I, \mathbb{R}),\|\cdot\|_{\infty}\right)$ of continuous $\mathbb{R}$-valued functions of $I$. In fact, $\mu \in\left(\mathcal{M}_{0}(I, \mathbb{R}),\|\cdot\|_{\mathbf{V}}\right)$ defines $\mu^{*} \in\left(\mathcal{C}(I, \mathbb{R}),\|\cdot\|_{\infty}\right)^{*}$ by

$$
\mu^{*}(f)=\int_{I} f(t) \mathrm{d} \mu(t), \quad f \in \mathcal{C}(I, \mathbb{R}),
$$

which refers to the Riemann-Stieltjes integral (or Lebesgue-Stieltjes integral). Moreover, one has

$$
\|\mu\|_{\mathbf{V}}=\mathbf{V}(\mu, I)=\sup \left\{\int_{I} f \mathrm{~d} \mu: f \in \mathcal{C}(I, \mathbb{R}),\|f\|_{\infty}=1\right\} .
$$

For the general theory of the Riemann-Stieltjes integral and the Lebesgue-Stieltjes integral, see, e.g., 1 .

Very recently, the authors have established in 14 some basic theory for eigenvalues of the second order linear measure differential equations

$$
\mathrm{d} \dot{y}+\lambda y \mathrm{~d} t+y \mathrm{~d} \mu(t)=0, \quad t \in I .
$$

The Dirichlet boundary condition is

$$
y(0)=y(1)=0,
$$

while the Neumann boundary condition is

$$
\dot{y}(0)=\stackrel{\bullet}{y}(1)=0 .
$$

Definition 1.1. By a solution $y(t)$ to the boundary value problem (1.3), we mean that

- $y \in \mathcal{C}(I, \mathbb{R})$ fulfills the boundary condition (1.4) or (1.5),

- there exists a function $z: I \rightarrow \mathbb{R}$ such that the equations (containing the Lebesgue-Stieltjes integral)

$$
\begin{aligned}
& y(t)=y_{0}+\int_{[0, t]} z(s) \mathrm{d} s, \quad t \in[0,1], \\
& z(t)= \begin{cases}z_{0}, & t=0, \\
z_{0}-\int_{[0, t]} y(s) \mathrm{d} \mu(s), & t \in(0,1],\end{cases}
\end{aligned}
$$

are satisfied and then $\dot{y}(t):=z(t)$. Here $\left(y_{0}, z_{0}\right) \in \mathbb{R}^{2}$. 
Definition 1.2. Given $\mu \in \mathcal{M}_{0}(I, \mathbb{R})$, we say that $\lambda \in \mathbb{C}$ is an eigenvalue of the Dirichlet problem (1.3)-(1.4), if equation (1.3) with such a parameter $\lambda$ has non-zero solutions $y(t)$ satisfying (1.4). The corresponding solutions $y(t)$ are called eigenfunctions associated with $\lambda$. The eigenvalues and eigenfunctions for the Neumann problem (1.3)-(1.5) are defined similarly.

Let us recall some known facts about eigenvalue theory of the second order measure differential equations ([14).

$\left(\mathbf{f}_{1}\right)$ Problem (1.3)-(1.4) has a sequence of real (simple) eigenvalues $\lambda_{m}^{D}(\mu), m \in$ $\mathbb{N}$, increasing in $m \in \mathbb{N}$.

$\left(\mathbf{f}_{2}\right)$ Problem (1.3)-(1.5) has a sequence of real (simple) eigenvalues $\lambda_{m}^{N}(\mu), m \in$ $\mathbb{Z}^{+}$, increasing in $m \in \mathbb{Z}^{+}$. Here $\mathbb{Z}^{+}:=\{0\} \cup \mathbb{N}$.

$\left(\mathbf{f}_{3}\right)$ Moreover, $\lim _{m \rightarrow \infty} \lambda_{m}^{D}(\mu)=\lim _{m \rightarrow \infty} \lambda_{m}^{N}(\mu)=+\infty$.

For $r \in(0, \infty)$, denote

$$
B_{0}[r]:=\left\{\mu \in \mathcal{M}_{0}(I, \mathbb{R}):\|\mu\|_{\mathbf{v}} \leq r\right\} .
$$

In this paper we will study the following minimization and maximization problems:

$$
\mathbf{L}_{m}(r):=\inf _{q \in B_{0}[r]} \lambda_{m}^{\sigma}(q) \quad \text { and } \quad \mathbf{M}_{m}(r):=\sup _{q \in B_{0}[r]} \lambda_{m}^{\sigma}(q) .
$$

Here $m \in \mathbb{N}$ for $\sigma=D$ or $m \in \mathbb{Z}^{+}$for $\sigma=N$. Due to the relation between the Dirichlet and Neumann eigenvalues, for $m \in \mathbb{N}$, both the Dirichlet and Neumann eigenvalues will result in the same infimum and supremum.

The main results of this paper are as follows.

Theorem 1.3. Define

$$
\begin{aligned}
& \hat{\mathbf{Z}}(x)=\sqrt{-x} \tanh \sqrt{-x} \quad \text { for } x \in(-\infty, 0] \text {, } \\
& \mathbf{Z}(x)= \begin{cases}2 \sqrt{-x} \operatorname{coth}(\sqrt{-x} / 2) & \text { for } x \in(-\infty, 0), \\
4 & \text { for } x=0, \\
2 \sqrt{x} \cot (\sqrt{x} / 2) & \text { for } x \in\left(0, \pi^{2}\right],\end{cases} \\
& \mathbf{Y}(x)=\left(\pi+\sqrt{\pi^{2}+4 x}\right)^{2} / 4 \quad \text { for } x \in[0, \infty) \text {. }
\end{aligned}
$$

Then $\hat{\mathbf{Z}}:(-\infty, 0] \rightarrow[0, \infty), \mathbf{Z}:\left(-\infty, \pi^{2}\right] \rightarrow[0, \infty), \mathbf{Y}:[0, \infty) \rightarrow\left[\pi^{2}, \infty\right)$ and

$$
\begin{aligned}
\mathbf{L}_{0}(r) & =\hat{\mathbf{Z}}^{-1}(r), \\
\mathbf{M}_{0}(r) & =r \\
\mathbf{L}_{m}(r) & =m^{2} \mathbf{Z}^{-1}\left(r / m^{2}\right) \quad \text { for } m \in \mathbb{N}, \\
\mathbf{M}_{m}(r) & =m^{2} \mathbf{Y}\left(r / m^{2}\right) \quad \text { for } m \in \mathbb{N},
\end{aligned}
$$

hold for each $r>0$.

The physical explanation to Theorem 1.3 is as follows. If a string has the total mass $r>0$, then the $m$-th Dirichlet/Neumann frequency can change in the interval $\left[\mathbf{L}_{m}(r), \mathbf{M}_{m}(r)\right]$, regardless of the distribution of its mass.

This paper is organized as follows. In Section 2, after recalling some basic facts on measures and topologies, we will prove a simplified version of the Alexandroff Theorem for the weak* convergence in the one-dimensional case. Then, we will obtain the approximation of general measures by smooth measures in weak ${ }^{*}$ topology. In Section 3, we will use the approximation of general measures and the continuity results of eigenvalues to solve extremal problems for eigenvalues of measure differential equations. 


\section{Auxiliary lemmas}

For the convenience of the reader, we present here some necessary basic facts to be used in this paper.

Let $I=[0,1]$. For any subinterval $I_{0} \subset I$, closed, open or semi-open, the total variation of $\mu$ over $I_{0}$ is also well defined. For example, if $I_{0}=(a, b] \subset I$, the total variation is

$\mathbf{V}\left(\mu, I_{0}\right):=\sup \left\{\sum_{i=0}^{n-1}\left|\mu\left(t_{i+1}\right)-\mu\left(t_{i}\right)\right|: a<t_{0}<t_{1}<\cdots<t_{n-1}<t_{n}=b, n \in \mathbb{N}\right\}$.

Recall the space $\mathcal{M}_{0}(I, \mathbb{R})$ is as in (1.1). Note that the normalization condition for $\mu \in \mathcal{M}_{0}(I, \mathbb{R})$ is $\mu(0+)=0$. Hence $\mu(0) \neq 0$ is possible. Due to the right-continuity of $\mu \in \mathcal{M}_{0}(I, \mathbb{R})$ on $(0,1)$, one has the following result for variations:

$$
\lim _{t \downarrow t_{0}} \mathbf{V}\left(\mu,\left[t_{0}, t\right]\right)=\lim _{t \downarrow t_{0}} \mathbf{V}\left(\mu,\left(t_{0}, t\right]\right)=0, \quad t_{0} \in(0,1) .
$$

Given $\mu \in \mathcal{M}_{0}(I, \mathbb{R})$ and $f \in \mathcal{C}(I, \mathbb{R})$, for any subinterval $I_{0} \subset I$, closed, open or semi-open, the Lebesgue-Stieltjes integral $\int_{I_{0}} f \mathrm{~d} \mu$ is also defined. Due to the jump of a measure $\mu(t)$ at $t=0$, one has

$$
\int_{[0, b]} f \mathrm{~d} \mu=-f(0) \mu(0)+\int_{(0, b]} f \mathrm{~d} \mu, \quad b \in(0,1] .
$$

That is, $\int_{[0, b]} f \mathrm{~d} \mu$ and $\int_{(0, b]} f \mathrm{~d} \mu$ may differ. If $I_{0}$ has the form $(a, b),(a, b]$, where $0 \leq a<b \leq 1$, or the form $[0, b),[0, b]$, where $0<b \leq 1$, one has the following basic inequality:

$$
\left|\int_{I_{0}} f \mathrm{~d} \mu\right| \leq\|f\|_{\infty, I_{0}} \cdot \mathbf{V}\left(f, I_{0}\right), \quad\|f\|_{\infty, I_{0}}:=\sup _{t \in I_{0}}|f(t)| .
$$

We say that measures $\mu_{2} \geq \mu_{1}$ hold for measures $\mu_{1}, \mu_{2}$ if

$$
\int_{I} f(t) \mathrm{d} \mu_{2}(t) \geq \int_{I} f(t) \mathrm{d} \mu_{1}(t) \quad \text { for all } f \in \mathcal{C}_{+}:=\{f \in \mathcal{C}(I, \mathbb{R}): f(t) \geq 0, t \in I\} .
$$

In fact, if $\mu_{2} \geq \mu_{1}$, then $\mu_{2}-\mu_{1}$ is nondeceasing on $I$.

In the space $\mathcal{M}_{0}(I, \mathbb{R})$ of measures, one has the usual topology induced by the norm $\|\cdot\|_{\mathbf{v}}$. It holds that $\left(\mathcal{M}_{0}(I, \mathbb{R}),\|\cdot\|_{\mathbf{v}}\right)$ is a Banach space. Due to the duality relation (1.2), one has the following weak* topology $w^{*}$.

Definition 2.1. Let $\mu_{0}, \mu_{n} \in \mathcal{M}_{0}(I, \mathbb{R}), n \in \mathbb{N}$. We say that $\mu_{n}$ is weakly* convergent to $\mu_{0}$ if, for each $f \in \mathcal{C}(I, \mathbb{R})$, one has

$$
\lim _{n \rightarrow \infty} \int_{I} f(t) \mathrm{d} \mu_{n}(t)=\int_{I} f(t) \mathrm{d} \mu_{0}(t) .
$$

We remark that in some literature, this topology is just called the weak topology for measures. For general theory on weak topologies and weak* topologies, we refer to [3, 11. By the Banach-Alaoglu Theorem [11, pp. 229-230], we have the following simple characterization on relatively sequentially compact subsets of $\left(\mathcal{M}_{0}(I, \mathbb{R}), w^{*}\right)$.

Lemma 2.2. A subset $V \subset\left(\mathcal{M}_{0}(I, \mathbb{R}), w^{*}\right)$ is relatively sequentially compact iff $V$ is bounded in the $\|\cdot\|_{\mathbf{V}}$ norm. That is, any sequence $\left\{\mu_{n}\right\} \subset \mathcal{M}_{0}(I, \mathbb{R})$ with $\sup _{n}\left\|\mu_{n}\right\|_{\mathbf{v}}<\infty$ has a subsequence converging to some $\mu_{0}$ in the space $\left(\mathcal{M}_{0}(I, \mathbb{R}), w^{*}\right)$. 
Let us recall the Alexandroff Theorem [3, p. 316] for weak* convergence.

Lemma 2.3. Let $\mu_{n}, \mu_{0} \in \mathcal{M}_{0}(I, \mathbb{R})$. Suppose that

- $\left\{\left\|\mu_{n}\right\|_{\mathbf{V}}\right\}_{n \in \mathbb{N}}$ is bounded, and

- for any open subset $B \subset I$ satisfying $\mu_{0}(B)=\mu_{0}(\bar{B})$, one has $\mu_{n}(B) \rightarrow \mu_{0}(B)$. Then one has $\mu_{n} \rightarrow \mu_{0}$ in $\left(\mathcal{M}_{0}(I, \mathbb{R}), w^{*}\right)$.

The following is a simplified version of the Alexandroff Theorem in the onedimensional case.

Lemma 2.4. Let $\mu_{n}, \mu_{0} \in \mathcal{M}_{0}(I, \mathbb{R})$. Suppose that

- $\left\{\left\|\mu_{n}\right\|_{\mathbf{v}}\right\}_{n \in \mathbb{N}}$ is bounded, and

- there holds

$$
\lim _{n \rightarrow \infty}\left(\mu_{n}(t)-\mu_{n}(0)\right)=\mu_{0}(t)-\mu_{0}(0) \quad \forall t \in S_{\mu_{0}},
$$

where

$$
S_{\mu_{0}}:=\left\{s \in(0,1]: \text { either } s=1 \text { or } s \in(0,1) \text { so that } \mu_{0}(t) \text { is continuous at } s\right\} \text {. }
$$

Then $\mu_{n} \rightarrow \mu_{0}$ in $\left(\mathcal{M}_{0}(I, \mathbb{R}), w^{*}\right)$.

Proof. Let $f \in \mathcal{C}(I, \mathbb{R})$ be given. For each $\varepsilon>0$, there exists $\delta>0$ such that

$$
t, s \in[0,1],|t-s|<\delta \Longrightarrow|f(t)-f(s)|<\varepsilon .
$$

It is well known that each measure on $I$ admits at most a countable set of discontinuity points. Hence we are able to choose $0=t_{0}<t_{1}<\cdots<t_{k}=1$ such that

$$
\left|t_{i+1}-t_{i}\right|<\delta, \quad i=0, \cdots, k-1,
$$

and all $\mu_{n}(t), n \in \mathbb{Z}^{+}$, are continuous at $t_{i}, i=1, \cdots, k-1$, i.e.,

$$
t_{1}, t_{2}, \cdots, t_{k} \in \bigcap_{n \in \mathbb{Z}^{+}} S_{\mu_{n}} .
$$

Here $\mathbb{Z}^{+}:=\{0\} \cup \mathbb{N}$. Define the step function

$$
f_{\varepsilon}(t):=\sum_{i=0}^{k-2} f\left(t_{i}\right) \cdot \chi_{\left[t_{i}, t_{i+1}\right)}(t)+f\left(t_{k-1}\right) \cdot \chi_{\left[t_{k-1}, t_{k}\right]}(t), \quad t \in I,
$$

where $\chi_{A}$ is the characteristic function of set $A$. Then one has

$$
\left\|f_{\varepsilon}-f\right\|_{\infty}<\varepsilon .
$$

As $\mu_{n}(t)$ is continuous at $t=t_{1}, t_{2}, \cdots, t_{k-1}$, one has

$$
\begin{aligned}
\mu_{n}\left(\left[t_{i}, t_{i+1}\right)\right) & =\mu_{n}\left(t_{i+1}\right)-\mu_{n}\left(t_{i}\right) \quad \text { for } i=0, \cdots, k-2, \\
\mu_{n}\left(\left[t_{k-1}, t_{k}\right]\right) & =\mu_{n}\left(t_{k}\right)-\mu_{n}\left(t_{k-1}\right),
\end{aligned}
$$

where $n \in \mathbb{Z}^{+}$. Thus,

$$
\begin{aligned}
\int_{I} f_{\varepsilon}(t) \mathrm{d} \mu_{n}(t) & =\sum_{i=0}^{k-2} \int_{\left[t_{i}, t_{i+1}\right)} f_{\varepsilon}(t) \mathrm{d} \mu_{n}(t)+\int_{\left[t_{k-1}, t_{k}\right]} f_{\varepsilon}(t) \mathrm{d} \mu_{n}(t) \\
& =\sum_{i=0}^{k-2} f\left(t_{i}\right)\left(\mu_{n}\left(t_{i+1}\right)-\mu_{n}\left(t_{i}\right)\right)+f\left(t_{k-1}\right)\left(\mu_{n}\left(t_{k}\right)-\mu_{n}\left(t_{k-1}\right)\right) .
\end{aligned}
$$


Note that

$$
\mu_{n}\left(t_{i+1}\right)-\mu_{n}\left(t_{i}\right) \equiv\left(\mu_{n}\left(t_{i+1}\right)-\mu_{n}(0)\right)-\left(\mu_{n}\left(t_{i}\right)-\mu_{n}(0)\right),
$$

where $t_{1}, t_{2}, \cdots, t_{k} \in S_{\mu_{0}}$. It follows from assumption (2.4) that

$$
\begin{aligned}
\lim _{n \rightarrow \infty} \int_{I} f_{\varepsilon}(t) \mathrm{d} \mu_{n}(t) & =\sum_{i=0}^{k-2} f\left(t_{i}\right)\left(\mu_{0}\left(t_{i+1}\right)-\mu_{0}\left(t_{i}\right)\right)+f\left(t_{k-1}\right)\left(\mu_{0}\left(t_{k}\right)-\mu_{0}\left(t_{k-1}\right)\right) \\
& =\int_{I} f_{\varepsilon}(t) \mathrm{d} \mu_{0}(t) .
\end{aligned}
$$

Let $K>0$ be such that $\left\|\mu_{n}\right\|_{\mathbf{v}} \leq K$ for all $n \in \mathbb{Z}^{+}$. Then

$$
\begin{aligned}
& \left|\int_{I} f(t) \mathrm{d} \mu_{n}(t)-\int_{I} f(t) \mathrm{d} \mu_{0}(t)\right| \\
\leq & \left|\int_{I}\left(f(t)-f_{\varepsilon}(t)\right) \mathrm{d}\left(\mu_{n}(t)-\mu_{0}(t)\right)\right|+\left|\int_{I} f_{\varepsilon}(t) \mathrm{d}\left(\mu_{n}(t)-\mu_{0}(t)\right)\right| \\
\leq & 2 K \varepsilon+\left|\int_{I} f_{\varepsilon}(t) \mathrm{d}\left(\mu_{n}(t)-\mu_{0}(t)\right)\right| .
\end{aligned}
$$

Since $\varepsilon$ is arbitrary, by using (2.5), we obtain

$$
\lim _{n \rightarrow \infty} \int_{I} f(t) \mathrm{d} \mu_{n}(t)=\int_{I} f(t) \mathrm{d} \mu_{0}(t) .
$$

Therefore $\mu_{n} \rightarrow \mu_{0}$ in $\left(\mathcal{M}_{0}(I, \mathbb{R}), w^{*}\right)$.

In this section, we will consider the approximation of general measures by smooth measures on $I$. We first need the following lemma.

Lemma 2.5. Given $\mu_{0} \in \mathcal{M}_{0}(I, \mathbb{R})$, there exists a sequence of measures $\left\{\nu_{n}\right\} \subset$ $C^{\infty}(I, \mathbb{R})$ such that

$$
\nu_{n}(0)=\mu_{0}(0), \quad \nu_{n}(1)=\mu_{0}(1)
$$

and

$\lim _{n \rightarrow \infty} \nu_{n}(t)=\mu_{0}(t) \quad$ for each point $t \in(0,1)$ at which $\mu_{0}(\cdot)$ is continuous.

Proof. Let

$$
\beta(t):= \begin{cases}e^{\frac{1}{|t|^{2}-1}} & \text { for }|t|<1 \\ 0 & \text { for }|t| \geq 1\end{cases}
$$

and

$$
\alpha(t):=\frac{\beta(t)}{\int_{\mathbb{R}} \beta(s) \mathrm{d} s}, \quad t \in \mathbb{R} .
$$

For $n \in \mathbb{N}$, define $\alpha_{n}(t)=n \alpha(n t)$. So $\alpha_{n}(t)=0$ when $|t| \geq 1 / n$ and $\int_{\mathbb{R}} \alpha_{n}(s) \mathrm{d} s=1$. Let

$$
\tilde{\mu}(t):= \begin{cases}0 & \text { for } t<-1, \\ 2 \mu_{0}(0)-\mu_{0}(-t) & \text { for } t \in[-1,0), \\ \mu_{0}(t) & \text { for } t \in[0,1] \\ 2 \mu_{0}(1)-\mu_{0}(2-t) & \text { for } t \in(1,2] \\ 0 & \text { for } t>2\end{cases}
$$


Let us take

$$
\nu_{n}(t):=\int_{\mathbb{R}} \tilde{\mu}(t-s) \alpha_{n}(s) \mathrm{d} s=\int_{\mathbb{R}} \tilde{\mu}(s) \alpha_{n}(t-s) \mathrm{d} s, \quad t \in[0,1], \quad n \in \mathbb{N} .
$$

Then $\nu_{n}(t)$ is $C^{\infty}(I, \mathbb{R})$ since $\alpha_{n}(t)$ is $C^{\infty}(\mathbb{R}, \mathbb{R})$. Moreover, we have the following conditions:

(i) At $t=0$,

$$
\begin{aligned}
\nu_{n}(0) & =\int_{\mathbb{R}} \tilde{\mu}(-s) \alpha_{n}(s) \mathrm{d} s \\
& =\int_{\mathbb{R}}\left(\tilde{\mu}(-s)-\mu_{0}(0)\right) \alpha_{n}(s) \mathrm{d} s+\int_{\mathbb{R}} \mu_{0}(0) \alpha_{n}(s) \mathrm{d} s \\
& =\int_{[-1 / n, 1 / n]}\left(\tilde{\mu}(-s)-\mu_{0}(0)\right) \alpha_{n}(s) \mathrm{d} s+\int_{\mathbb{R}} \mu_{0}(0) \alpha_{n}(s) \mathrm{d} s \\
& =\mu_{0}(0),
\end{aligned}
$$

since $\tilde{\mu}(t)-\mu_{0}(0)=-\left(\tilde{\mu}(-t)-\mu_{0}(0)\right), \alpha_{n}(t)=\alpha_{n}(-t)$ for $t \in[-1,1]$ and $\int_{\mathbb{R}} \alpha_{n}(s) \mathrm{d} s$ $=1$.

(ii) At $t=1$,

$$
\begin{aligned}
\nu_{n}(1) & =\int_{\mathbb{R}} \tilde{\mu}(1-s) \alpha_{n}(s) \mathrm{d} s \\
& =\int_{\mathbb{R}}\left(\tilde{\mu}(1-s)-\mu_{0}(1)\right) \alpha_{n}(s) \mathrm{d} s+\int_{\mathbb{R}} \mu_{0}(1) \alpha_{n}(s) \mathrm{d} s \\
& =\int_{[-1 / n, 1 / n]}\left(\tilde{\mu}(1-s)-\mu_{0}(1)\right) \alpha_{n}(s) \mathrm{d} s+\int_{\mathbb{R}} \mu_{0}(1) \alpha_{n}(s) \mathrm{d} s \\
& =\mu_{0}(1),
\end{aligned}
$$

since $\tilde{\mu}(1-t)-\mu_{0}(1)=-\left(\tilde{\mu}(1+t)-\mu_{0}(1)\right)$ for $t \in[-1,1]$.

(iii) When $t \in(0,1)$ and $\mu_{0}$ is continuous at $t$,

$$
\begin{aligned}
\left|\nu_{n}(t)-\mu_{0}(t)\right| & =\left|\int_{\mathbb{R}}\left(\tilde{\mu}(t-s)-\mu_{0}(t)\right) \alpha_{n}(s) \mathrm{d} s\right| \\
& \leq \sup _{l \in[-1 / n, 1 / n]}\left|\tilde{\mu}(t-l)-\mu_{0}(t)\right| \int_{[-1 / n, 1 / n]} \alpha_{n}(s) \mathrm{d} s \\
& \rightarrow 0,
\end{aligned}
$$

as $n \rightarrow \infty$ since $\mu_{0}$ is continuous at $t \in(0,1)$.

Next, we will show that in general, a measure cannot be the limit point of smooth measures in the norm $\|\cdot\| \mathbf{v}$. However, in the $w^{*}$ topology, the following conclusion holds.

Lemma 2.6. Given $\mu_{0} \in \mathcal{M}_{0}(I, \mathbb{R})$, there exists a sequence of measures $\left\{\mu_{n}\right\} \subset$ $C^{\infty}(I, \mathbb{R}) \cap \mathcal{M}_{0}(I, \mathbb{R})$ such that

$$
\mu_{n} \rightarrow \mu_{0} \text { in }\left(\mathcal{M}_{0}(I, \mathbb{R}), w^{*}\right) .
$$

Moreover, if $\mu_{0}$ is increasing (decreasing) on $I$, then the sequence $\left\{\mu_{n}\right\}$ above can be chosen such that for $n \in \mathbb{N}, \mu_{n}$ is increasing (decreasing) on I and $\left\|\mu_{n}\right\|_{\mathbf{V}}=\left\|\mu_{0}\right\|_{\mathbf{V}}$.

Proof. Take

$$
\mu_{n}(t):=\nu_{n}(t)-\mu_{0}(0), \quad t \in[0,1], \quad n \in \mathbb{N},
$$


where $\nu_{n}(t)$ is as in (2.8). Then $\mu_{n}(t)$ is $C^{\infty}(I, \mathbb{R})$ since $\nu_{n}(t)$ is $C^{\infty}(I, \mathbb{R})$. Moreover, we have

$$
\mu_{n}(0+)=\mu_{n}(0)=\nu_{n}(0)-\mu_{0}(0)=0 .
$$

So $\mu_{n} \in \mathcal{M}_{0}(I, \mathbb{R}), n \in \mathbb{N}$.

We claim that the condition (2.4) in Lemma 2.4 holds. In fact, by Lemma 2.5. the following conditions hold:

(i) When $t=1$,

$$
\mu_{n}(1)-\mu_{n}(0)=\nu_{n}(1)-\mu_{0}(0)-\left(\nu_{n}(0)-\mu_{0}(0)\right)=\mu_{0}(1)-\mu_{0}(0) .
$$

(ii) When $t \in(0,1)$ and $\mu_{0}$ is continuous at $t$,

$$
\begin{aligned}
& \left|\left(\mu_{n}(t)-\mu_{n}(0)\right)-\left(\mu_{0}(t)-\mu_{0}(0)\right)\right|=\left|\left(\nu_{n}(t)-\nu_{n}(0)\right)-\left(\mu_{0}(t)-\mu_{0}(0)\right)\right| \\
= & \left|\nu_{n}(t)-\mu_{0}(t)\right| \rightarrow 0,
\end{aligned}
$$

as $n \rightarrow \infty$.

Next, we prove that the first condition in Lemma 2.4 also holds.

Since $\tilde{\mu}(t)$ has bounded total variation on $[-1,2]$, we can assume $\tilde{\mu}(t)=$ $f(t)-g(t)$, where $f$ and $g$ are increasing on $[-1,2]$. Hence for $t \in[0,1]$,

$$
\begin{aligned}
\mu_{n}(t) & =\nu_{n}(t)-\mu_{0}(0)=\int_{\mathbb{R}} f(t-s) \alpha_{n}(s) \mathrm{d} s-\int_{\mathbb{R}} g(t-s) \alpha_{n}(s) \mathrm{d} s-\mu_{0}(0) \\
& =: F_{n}(t)-G_{n}(t)-\mu_{0}(0),
\end{aligned}
$$

where $F_{n}(t)$ and $G_{n}(t)$ are increasing on $[0,1]$. Therefore

$$
\begin{aligned}
\left\|\mu_{n}\right\|_{\mathbf{V}} & \leq\left\|F_{n}\right\|_{\mathbf{V}}+\left\|G_{n}\right\|_{\mathbf{V}} \\
& =F_{n}(1)-F_{n}(0)+G_{n}(1)-G_{n}(0) \\
& =\int_{\mathbb{R}}(f(1-s)-f(-s)) \alpha_{n}(s) \mathrm{d} s+\int_{\mathbb{R}}(g(1-s)-g(-s)) \alpha_{n}(s) \mathrm{d} s \\
& \leq \int_{[-1 / n, 1 / n]}(|f(1-s)|+|f(-s)|+|g(1-s)|+|g(-s)|) \alpha_{n}(s) \mathrm{d} s \\
& \leq 2\left(\|f\|_{\infty,[-1,2]}+\|g\|_{\infty,[-1,2]}\right) \int_{[1 / n, 1 / n]} \alpha_{n}(s) \mathrm{d} s=\text { const. }
\end{aligned}
$$

By Lemma 2.4, we conclude that

$$
\mu_{n} \rightarrow \mu_{0} \quad \text { in }\left(\mathcal{M}_{0}(I, \mathbb{R}), w^{*}\right) .
$$

Moreover, if $\mu_{0}$ is increasing (decreasing) on $[0,1]$, then $\tilde{\mu}(t)$ is increasing (decreasing) on $[-1,2]$, which implies $\mu_{n}$ is also increasing (decreasing) on $[0,1]$. Hence by (2.10),

$$
\left\|\mu_{n}\right\|_{\mathbf{V}}=\left|\mu_{n}(1)-\mu_{n}(0)\right|=\left|\mu_{0}(1)-\mu_{0}(0)\right|=\left\|\mu_{0}\right\|_{\mathbf{V}}
$$

Remark 2.7. The result above shows that the subset $C^{\infty}(I, \mathbb{R}) \cap \mathcal{M}_{0}(I, \mathbb{R})$ is dense in $\mathcal{M}_{0}(I, \mathbb{R})$ with the weak* topology. However, we have that the subset $C^{\infty}(I, \mathbb{R}) \cap$ $\mathcal{M}_{0}(I, \mathbb{R})$ is not dense in $\mathcal{M}_{0}(I, \mathbb{R})$ with the $\|\cdot\|_{\mathrm{V}}$ topology since

$$
\overline{C^{\infty}(I, \mathbb{R}) \cap \mathcal{M}_{0}(I, \mathbb{R})} \subset \mathcal{C}(I, \mathbb{R}),
$$

where the closure is taken in $\left(\mathcal{M}_{0}(I, \mathbb{R}),\|\cdot\|_{\mathbf{V}}\right)$. 
Definition 2.8. For $\mu(t) \in \mathcal{M}_{0}(I, \mathbb{R})$, we say $\mu(t)$ has density if $\mu(t)$ is absolutely continuous with respect to the Lebesgue measure, i.e., there exists $q(t) \in \mathcal{L}^{1}(I, \mathbb{R})$ such that

$$
\frac{\mathrm{d} \mu(t)}{\mathrm{d} t}=q(t) \quad \text { a.e. } \quad t \in I .
$$

$q(t)$ is called the density of the measure $\mu(t)$.

The following conclusion is an immediate consequence of Lemma 2.6 .

Lemma 2.9. Let

$$
E=\left\{\nu(t) \in \mathcal{M}_{0}(I, \mathbb{R}): \nu(t) \text { has density }\right\} .
$$

Then $E$ is a dense subset in $\left(\mathcal{M}_{0}(I, \mathbb{R}), w^{*}\right)$. In other words, for each $\mu(t) \in$ $\mathcal{M}_{0}(I, \mathbb{R})$, there exists a sequence $\left\{q_{n}(t)\right\} \subset \mathcal{L}^{1}(I, \mathbb{R})$ such that

$$
\lim _{n \rightarrow \infty} \int_{I} f(t) q_{n}(t) \mathrm{d} t=\int_{I} f(t) \mathrm{d} \mu(t) \quad \forall f \in \mathcal{C}(I, \mathbb{R}) .
$$

\section{MAin Results}

In this section, we consider the eigenvalue problem (1.7). First, we will give some preliminary results on eigenvalues of the equation (1.3).

Theorem 3.1 ([14]). Let $m \in \mathbb{N}$ for $\sigma=D$ or $m \in \mathbb{Z}^{+}$for $\sigma=N$. Then

(i) If $\mu_{2} \geq \mu_{1}$, one has $\lambda_{m}^{\sigma}\left(\mu_{2}\right) \leq \lambda_{m}^{\sigma}\left(\mu_{1}\right)$.

(ii) In the weak* topology of measures, $\lambda_{m}^{\sigma}(\mu)$ is continuous in $\mu \in\left(\mathcal{M}_{0}(I, \mathbb{R}), w^{*}\right)$.

When the measure $\mu \in \mathcal{M}_{0}(I, \mathbb{R})$ is absolutely continuous with the density $q(t)=$ $\frac{\mathrm{d} \mu(t)}{\mathrm{d} t} \in \mathcal{L}^{1}(I, \mathbb{R}),(1.3)$ reduces to a classical Sturm-Liouville problem as follows:

$$
\ddot{y}+(\lambda+q(t)) y=0, \quad t \in I .
$$

In this case, we have $\lambda_{m}^{\sigma}(q)=\lambda_{m}^{\sigma}(\mu)$.

In recent works 19, 20, the authors have successfully solved several minimization and maximization problems concerning the eigenvalues of equation (3.1). For $r \in(0, \infty)$, let

$$
B_{1}[r]:=\left\{q \in \mathcal{L}^{1}(I, \mathbb{R}):\|q\|_{1} \leq r\right\}
$$

be the $L^{1}$ ball. Consider the following minimization and maximization problems:

$$
\tilde{\mathbf{L}}_{m}(r):=\inf _{q \in B_{1}[r]} \lambda_{m}^{\sigma}(q) \quad \text { and } \quad \tilde{\mathbf{M}}_{m}(r):=\sup _{q \in B_{1}[r]} \lambda_{m}^{\sigma}(q) .
$$

Due to the relation between the Dirichlet and Neumann eigenvalues [19], for $m \in \mathbb{N}$, both the Dirichlet and Neumann eigenvalues will result in the same infimum and supremum. The results in [19,20] are as follows.

Theorem $3.2([19,20])$. We have

$$
\begin{aligned}
\tilde{\mathbf{L}}_{0}(r) & =\hat{\mathbf{Z}}^{-1}(r), \\
\tilde{\mathbf{M}}_{0}(r) & =r \\
\tilde{\mathbf{L}}_{m}(r) & =m^{2} \mathbf{Z}^{-1}\left(r / m^{2}\right) \quad \text { for } m \in \mathbb{N}, \\
\tilde{\mathbf{M}}_{m}(r) & =m^{2} \mathbf{Y}\left(r / m^{2}\right) \quad \text { for } m \in \mathbb{N} .
\end{aligned}
$$

Here functions $\hat{\mathbf{Z}}, \mathbf{Z}$ and $\mathbf{Y}$ are as in (1.8), (1.9) and (1.10), respectively. 
Note that $B_{1}[r]$ has no compactness. To obtain these results, the authors have first solved the approximating minimization and maximization problem for eigenvalues on the corresponding $L^{p}$ balls, $1<p<\infty$. Then one can obtain results in Theorem 3.2 by letting $p \downarrow 1$.

As a consequence of continuity results in Theorem 3.1, the extremal values (1.7)

$$
\mathbf{L}_{m}(r)=\min _{\mu \in B_{0}[r]} \lambda_{m}^{\sigma}(\mu) \quad \text { and } \quad \mathbf{M}_{m}(r)=\max _{\mu \in B_{0}[r]} \lambda_{m}^{\sigma}(\mu)
$$

are finite and can be attained by some measures in $B_{0}[r]$, because $B_{0}[r]$ is sequentially compact in $\left(\mathcal{M}_{0}(I, \mathbb{R}), w^{*}\right)([3])$.

Now we are ready to prove the main theorem of this paper.

Proof of Theorem 1.3. First, we will prove that

$$
\mathbf{L}_{m}(r)=\tilde{\mathbf{L}}_{m}(r) \quad \text { and } \quad \mathbf{M}_{m}(r)=\tilde{\mathbf{M}}_{m}(r)
$$

for all $m \in \mathbb{Z}^{+}$.

Given $q(t) \in B_{1}[r]$, the measure $\mu_{q}(t) \in \mathcal{M}_{0}(I, \mathbb{R})$ is defined by

$$
\mu_{q}(t):=\int_{[0, t]} q(s) \mathrm{d} s .
$$

We have $\left\|\mu_{q}\right\|_{\mathbf{V}}=\|q\|_{1}$ and then $\mu_{q} \in B_{0}[r]$ is absolutely continuous with respect to the Lebesgue measure.

So for any $q \in B_{1}[r]$,

$$
\mathbf{L}_{m}(r) \leq \lambda_{m}^{\sigma}\left(\mu_{q}\right)=\lambda_{m}^{\sigma}(q)
$$

which implies that

$$
\mathbf{L}_{m}(r) \leq \tilde{\mathbf{L}}_{m}(r) .
$$

On the other hand, for each $m$, there exists $\bar{\mu} \in B_{0}[r]$ such that $\lambda_{m}^{\sigma}(\bar{\mu})=\mathbf{L}_{m}(r)$. From basic properties for eigenvalues like the monotonicity of $\lambda_{m}^{\sigma}(\mu)$ in $\mu([14])$, we have $\bar{\mu}$ is increasing. By Lemma 2.6. there exists a sequence of measures $\left\{\bar{\mu}_{n}\right\} \subset$ $C^{\infty}(I, \mathbb{R}) \cap \mathcal{M}_{0}(I, \mathbb{R})$ with $\frac{\mathrm{d} \bar{\mu}_{n}(t)}{\mathrm{d} t}=\bar{q}_{n}(t)$ such that

$$
\left\|\bar{\mu}_{n}\right\|_{\mathbf{V}}=\left\|\bar{q}_{n}\right\|_{1}=\|\bar{\mu}\|_{\mathbf{v}} \leq r
$$

and

$$
\bar{\mu}_{n} \rightarrow \bar{\mu} \text { in }\left(\mathcal{M}_{0}(I, \mathbb{R}), w^{*}\right) .
$$

Therefore, by Theorem 3.1, we have

$$
\mathbf{L}_{m}(r)=\lambda_{m}^{\sigma}(\bar{\mu})=\lim _{n \rightarrow \infty} \lambda_{m}^{\sigma}\left(\bar{\mu}_{n}\right)=\lim _{n \rightarrow \infty} \lambda_{m}^{\sigma}\left(\bar{q}_{n}\right) \geq \lim _{n \rightarrow \infty} \tilde{\mathbf{L}}_{m}(r)=\tilde{\mathbf{L}}_{m}(r) .
$$

(3.6) and (3.7) imply that $\mathbf{L}_{m}(r)=\tilde{\mathbf{L}}_{m}(r)$.

We can argue in a similar way to prove the maximization problem.

Hence, by Theorem 3.2 the proof is complete.

Remark 3.3. In [21], Zhang has studied the minimization problem $\mathbf{L}_{0}(r)$ as in (1.7) of the zeroth Neumann eigenvalues. Notice that the boundary $\partial B_{0}[r]$ is non-smooth in the Banach space $\left(\mathcal{M}_{0}(I, \mathbb{R}),\|\cdot\|_{\mathbf{v}}\right)$. By using the Lagrangian multiplier method for non-smooth functionals, Zhang has proved in [21] that

$$
\mathbf{L}_{0}(r)=\hat{\mathbf{Z}}^{-1}(r) .
$$

The results in Theorem 1.3 cover the main results in [21. However, the methods in this paper are totally different from those in [21]. In fact, we use some known facts 
on extremal eigenvalues of ODE and a new approximation result in this paper to give a simple method for extremal eigenvalues of measure differential equations.

\section{ACKNOWLEDGMENT}

The author would like to thank Ping Yan and Meirong Zhang for helpful discussions.

\section{REFERENCES}

[1] M. Carter and B. van Brunt, The Lebesgue-Stieltjes integral: A practical introduction, Undergraduate Texts in Mathematics, Springer-Verlag, New York, 2000. MR1759133 (2001k:26010)

[2] A. Derlet, J.-P. Gossez, and P. Takáč, Minimization of eigenvalues for a quasilinear elliptic Neumann problem with indefinite weight, J. Math. Anal. Appl. 371 (2010), no. 1, 69-79, DOI 10.1016/j.jmaa.2010.03.068. MR2660987 (2011h:49069)

[3] Nelson Dunford and Jacob T. Schwartz, Linear Operators. I. General Theory, With the assistance of W. G. Bade and R. G. Bartle. Pure and Applied Mathematics, Vol. 7, Interscience Publishers, Inc., New York, 1958. MR0117523 (22 \#8302)

[4] Jiří Jarník, Dependence of solutions of a class of differential equations of the second order on a parameter (English, with Russian summary), Czechoslovak Math. J. 15 (90) (1965), 124-160. MR0173053 (30 \#3268)

[5] Chiu-Yen Kao, Yuan Lou, and Eiji Yanagida, Principal eigenvalue for an elliptic problem with indefinite weight on cylindrical domains, Math. Biosci. Eng. 5 (2008), no. 2, 315-335, DOI 10.3934/mbe.2008.5.315. MR2494032(2010h:35282)

[6] Samir Karaa, Sharp estimates for the eigenvalues of some differential equations, SIAM J. Math. Anal. 29 (1998), no. 5, 1279-1300 (electronic), DOI 10.1137/S0036141096307849. MR $1628259(99 \mathrm{~d}: 34162)$

[7] M. G. Krein, On certain problems on the maximum and minimum of characteristic values and on the Lyapunov zones of stability, Amer. Math. Soc. Transl. (2) 1 (1955), 163-187. MR0073776 (17,484e)

[8] Jaroslav Kurzweil, Generalized ordinary differential equations and continuous dependence on a parameter (Russian), Czechoslovak Math. J. 7 (82) (1957), 418-449. MR0111875 (22 \#2735)

[9] Jaroslav Kurzweil, Generalized ordinary differential equations (English, with Russian summary), Czechoslovak Math. J. 8 (83) (1958), 360-388. MR0111878 (22 \#2738)

[10] Yuan Lou and Eiji Yanagida, Minimization of the principal eigenvalue for an elliptic boundary value problem with indefinite weight, and applications to population dynamics, Japan J. Indust. Appl. Math. 23 (2006), no. 3, 275-292. MR2281509 (2008i:35044)

[11] Robert E. Megginson, An introduction to Banach space theory, Graduate Texts in Mathematics, vol. 183, Springer-Verlag, New York, 1998. MR1650235 (99k:46002)

[12] Gang Meng, Ping Yan, and Meirong Zhang, Minimization of eigenvalues of one-dimensional p-Laplacian with integrable potentials, J. Optim. Theory Appl. 156 (2013), no. 2, 294-319, DOI 10.1007/s10957-012-0125-3. MR3019316

[13] Gang Meng, Ping Yan, and Meirong Zhang, Maximization of eigenvalues of one-dimensional p-Laplacian with integrable potentials, Commun. Contemp. Math. 15 (2013), no. 1, 1250049, 18, DOI 10.1142/S0219199712500496. MR3022996

[14] Gang Meng and Meirong Zhang, Dependence of solutions and eigenvalues of measure differential equations on measures, J. Differential Equations 254 (2013), no. 5, 2196-2232, DOI 10.1016/j.jde.2012.12.001. MR3007109

[15] Angelo B. Mingarelli, Volterra-Stieltjes integral equations and generalized ordinary differential expressions, Lecture Notes in Mathematics, vol. 989, Springer-Verlag, Berlin, 1983. MR706255 (86e:45001)

[16] Štefan Schwabik, Generalized ordinary differential equations, Series in Real Analysis, vol. 5, World Scientific Publishing Co. Inc., River Edge, NJ, 1992. MR.1200241 (94g:34004)

[17] Štefan Schwabik, Milan Tvrdý, and Otto Vejvoda, Differential and integral equations: Boundary value problems and adjoints, D. Reidel Publishing Co., Dordrecht, 1979. MR542283 (80h:34003) 
[18] M. Tvrdý, Differential and integral equations in the space of regulated functions (English, with English and Georgian summaries), Mem. Differential Equations Math. Phys. 25 (2002), 1-104. MR.1903190 (2003k:34025)

[19] Qiaoling Wei, Gang Meng, and Meirong Zhang, Extremal values of eigenvalues of SturmLiouville operators with potentials in $L^{1}$ balls, J. Differential Equations 247 (2009), no. 2, 364-400, DOI 10.1016/j.jde.2009.04.008. MR.2523683 (2010d:34212)

[20] Meirong Zhang, Extremal values of smallest eigenvalues of Hill's operators with potentials in $L^{1}$ balls, J. Differential Equations 246 (2009), no. 11, 4188-4220, DOI 10.1016/j.jde.2009.03.016. MR2517767 (2010d:34213)

[21] MeiRong Zhang, Extremal eigenvalues of measure differential equations with fixed variation, Sci. China Math. 53 (2010), no. 10, 2573-2588, DOI 10.1007/s11425-010-4081-9. MR2728263 (2012b:34248)

[22] Meirong Zhang, Minimization of the zeroth Neumann eigenvalues with integrable potentials, Ann. Inst. H. Poincaré Anal. Non Linéaire 29 (2012), no. 4, 501-523, DOI 10.1016/j.anihpc.2012.01.007. MR2948286

School of Mathematical Sciences, University of Chinese Academy of Sciences, BeiJiNG 100049, PeOple's Republic of ChinA

E-mail address: menggang@ucas.ac.cn 